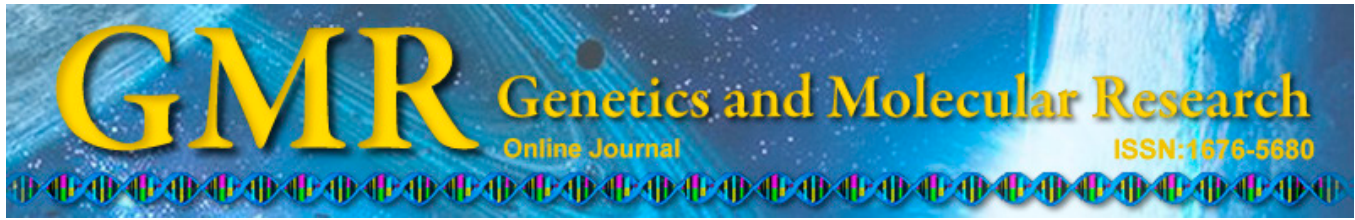

\title{
Erlotinib enhances the CIK cell-killing sensitivity of lung adenocarcinoma A549 cells
}

\author{
J.Z. Mei, G.J. Liu, X.J. Zhang, J.Z. Zhao and R.T. Feng \\ Department of Oncology, Zhengzhou Peoples Hospital, Zhengzhou, China \\ Corresponding author: J.Z. Mei \\ E-mail: meijiazhuan_mjz@yeah.net
}

Genet. Mol. Res. 14 (2): 3082-3089 (2015)

Received May 22, 2014

Accepted October 21, 2014

Published April 10, 2015

DOI http://dx.doi.org/10.4238/2015.April.10.18

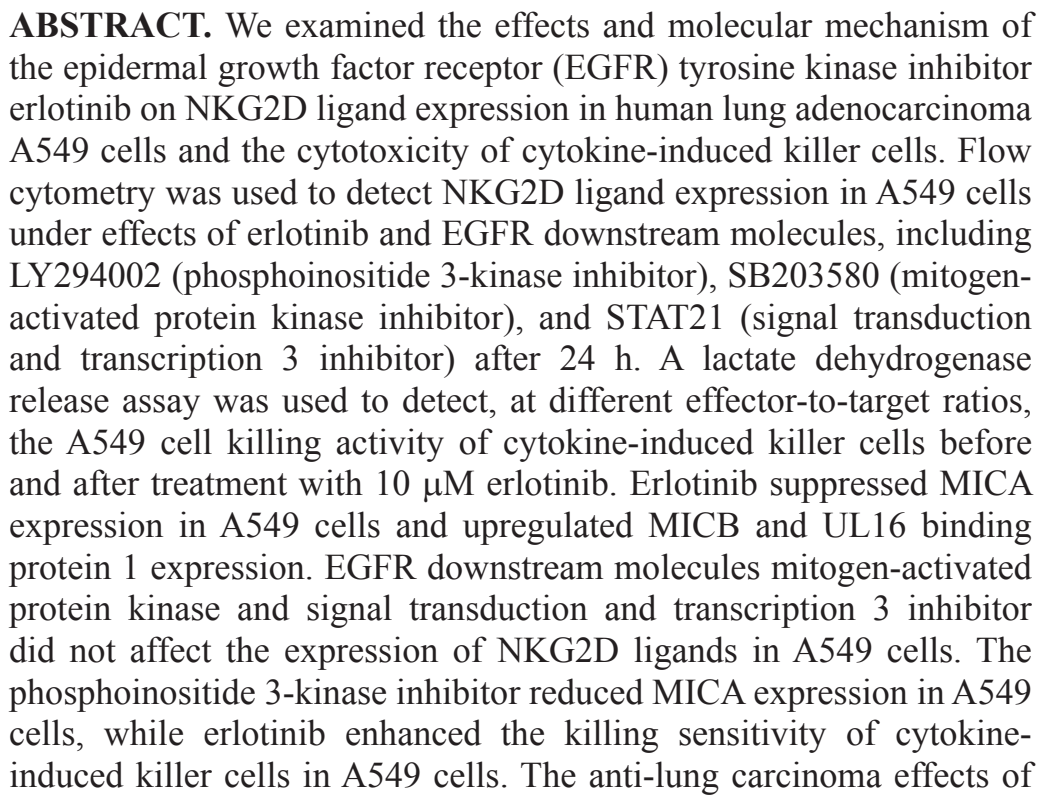


EGFR tyrosine kinase inhibitor were associated with the sensitivity of lung cancer cells to enhanced immune cell killing.

Key words: Cytokine-induced killer cells; NKG2D ligands; EGFR tyrosine kinase inhibitor; Lung cancer

\section{INTRODUCTION}

Chemotherapy and targeted drugs are the principal means of treating advanced nonsmall cell lung cancer. For patients with epidermal growth factor receptor (EGFR) mutations, EGFR tyrosine kinase inhibitors (EGFR TKI) can prolong the survival time of patients. Unfortunately, in the Asian population, EGFR mutations account for only 20-30\% of advanced non-small cell lung cancerin patients (Han et al., 2005). Determining an effective population to further develop the efficacy of EGFR TKI requires further study. The surface expression of the NKG2D ligands on tumor cells results in an attack targeted against the body's immune cells (Bae et al., 2012a). Previous studies (Mei et al., 2009; Inagaki et al., 2009; Morisaki et al., 2011; Huang et al., 2011) have shown that chemotherapy and targeted drug therapy can induce the surface expression of NKG2D ligands on tumor cells, which can enhance the killing activity of immune cells and improve anti-tumor effects. However, the myelosuppression of conventional chemotherapeutic drugs has limited the combination of chemotherapy and immune cell therapy. In a previous study, investigating the effects of the EGFR TKI erlotinib on NKG2D ligand expression on the surface of A549 cells and cytotoxicity of cytokine-induced killer (CIK) cells, human lung adenocarcinoma A549 cells were found to express wild-type EGFR (Rho et al., 2011). These results provide a theoretical and experimental basis for the interaction between erlotinib and immune cells in the treatment of lung cancer.

\section{MATERIAL AND METHODS}

\section{Materials}

The following materials were used in this study: recombinant human interleukin-2 (Liaoning Weixing Biological Products Institute, Liaoning, China); interferon- $\gamma$ (Shanghai Clone Bio-Tech Co., Ltd., Shanghai, China); lymphocyte separation medium (Tianjin Haoyang Biological Products Co., Ltd., Tianjin, China); RPMI 1640 (Gibco, Grand Island, NY, USA); tetrazolium blue (Sigma, St. Louis, MO, USA); CD3 mAb (ProSpec-Tany TechnoGene, Rehovot, Israel); FITC-CD4/PE-CD8/PerCP-CD3, FITC-CD56, PE-CD3, PerCP-CD3, NKG2D-PE, FITC-IgG1, PE-IgG1, MICA, MICB, ULBP1, ULBP2, ULBP3 monoclonal antibodies, and flow cytometry apparatus (BD Biosciences, Franklin Lakes, NJ, USA); lactate dehydrogenase release assay kit (Promega Corporation, Madison, WI, USA); erlotinib (Roche, Basel, Switzerland), LY294002, SB203580 (Sigma), and signal transduction and transcription 21 (STAT21) (Biomol, Farmingdale, NY, USA). The drugs were dissolved in dimethyl sulfoxide and stored at $-20^{\circ} \mathrm{C}$. Drugs were thawed before use. The RPMI 1640 culture medium containing $10 \%$ fetal bovine serum was diluted to the desired concentration. The final concentration of dimethyl sulfoxide was $<0.1 \%$. Human lung adenocarcinoma A549 cells were routinely passaged and stored in our center. 


\section{Methods}

\section{CIK cell preparation}

CIK cells were prepared according to the methods described in a previous study (Linn et al., 2012) and cultured for 14 days. Flow cytometry was used to detect the CIK cell phenotype.

\section{A549 cell culture}

A549 cells were seeded into 100-mL flasks. The cell culture medium was RPMI 1640 containing $10 \%$ fetal bovine serum, $100 \mathrm{U} / \mathrm{mL}$ penicillin, and $100 \mu \mathrm{g} / \mathrm{mL}$ streptomycin. The culture was placed in a $5 \% \mathrm{CO}_{2}$ incubator at $37^{\circ} \mathrm{C}$ for incubation. Cells were cultured to cover $70-80 \%$ of the flasks. Trypsin was used for digestion, counting, and passage.

\section{Flow cytometry analysis of NKG2D ligand expression in A549 cells before and after adding erlotinib}

A549 cells in logarithmic growth phase were seeded in 100-mL flasks at a concentration of $5 \times 10^{4} / \mathrm{mL}$. After $24 \mathrm{~h}$ of culture, erlotinib was added. When the final concentrations were equivalent to 5 and $10 \mu \mathrm{M}$, the empty drug group was used as the control group. After a 24-h culture, the A549 cells before and after the use of erlotinib were collected and washed with phosphate-buffered saline. The cells were counted and placed into different tubes. At a cell concentration of $1 \mu \mathrm{g} / 10^{6}$, MICA, MICB, ULBP1, ULBP2, and ULBP3 monoclonal antibodies (mAbs) were added at $4^{\circ} \mathrm{C}$ for 30 min. After washing with phosphate-buffered saline, the fluorescein isothiocyanate-labeled goat anti-mouse IgG1 secondary antibody was added at $4^{\circ} \mathrm{C}$ for $30 \mathrm{~min}$. After phosphate-buffered saline washing, the sample was analyzed. The same IgG1 antibody was used as a negative control. Flow cytometry was used to analyze positive cells among $1 \times 10^{4}$ cells, and the percentages were calculated. These experiments were repeated in triplicate

\section{Effects of downstream molecules of epidermal growth factor signaling pathways on NKG2D ligand expression in A549 cells using flow cytometry}

A549 cells in logarithmic growth phase were seeded in 100-mL flasks at a concentration of $5 \times 10^{4} / \mathrm{mL}$. After $24 \mathrm{~h}$ of culture, $30 \mu \mathrm{M}$ STAT21, $15 \mu \mathrm{M}$ LY294002, and $25 \mu \mathrm{M}$ SB203580 were added. The empty drug group was used as a control. After a 24-h culture, the A549 cells were collected. Flow cytometry was used to detect NKG2D ligand expression on the surface of A549 cells.

\section{CIK cell-killing activity}

A 4-h lactate dehydrogenase release assay was conducted (Mei et al., 2007) according to Cytotox 96 Non-Radioactive Cytotoxicity Assay instructions. CIK cell-killing activity before and after the use of erlotinib at a concentration of $10 \mu \mathrm{M}$ in A549 cells was observed in different effector to target ratios. When the effector target ratio was 20:1, NKG2D mAb was incubated with CIK cells for 15 min. A549 cells, before and after the use of erlotinib, at a con- 
centration of $10 \mu \mathrm{M}$, were added to measure the killing rate. The experiment was repeated 3 times.

\section{Statistical analysis}

The SPSS10.0 statistical software was used for analysis (SPSS, Inc., Chicago, IL, USA). Data are reported as means \pm standard deviation. The NKG2D ligand expression rate on the surface of A549 cells and the CIK cell-killing rate before and after drug treatment were compared using the paired sample $t$-test. In different effector target ratios, the cytotoxicity of CIK cells between groups was compared using one-way analysis of variance, with $\mathrm{P}<0.05$ indicating statistical significance.

\section{RESULTS}

\section{CIK cell phenotype}

Figure 1 shows the flow cytometry results: $\mathrm{CD} 3+>90 \%, \mathrm{CD} 3+\mathrm{CD} 8+>80 \%$, $\mathrm{CD} 3+\mathrm{CD} 56+>30 \%, \mathrm{CD} 3+\mathrm{CD} 4+<25 \%, \mathrm{NKG} 2 \mathrm{D}>80 \%$.
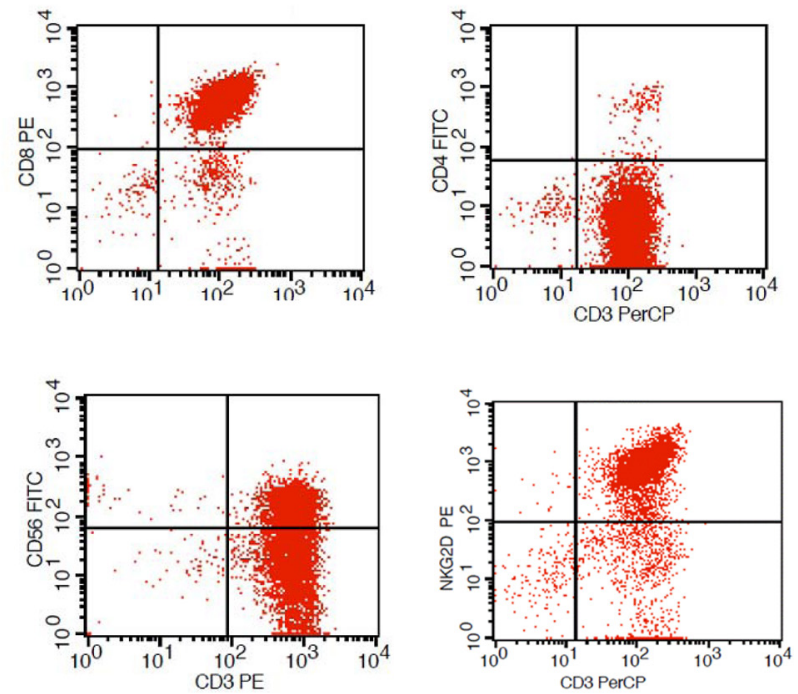

Figure 1. Phenotype of CIK cells.

\section{Erlotinib regulated expression of NKG2D ligands in A549 cells}

Figure 2 shows NKG2D ligand expression in A549 cells prior to treatment with erlotinib, which were as follows (blue curve represents the isotype control $\mathrm{mAb}$; red curve represents the original expression): MICA $66.62 \pm 1.42 \%$, MICB $32.75 \pm 2.37 \%$, ULBP1 $9.80 \pm$ $2.40 \%$, ULBP2 $4.09 \pm 1.94 \%$, and ULBP3 $34.96 \pm 4.22 \%$. Erlotinib at $5 \mu \mathrm{M}$ (green curve) and $10 \mu \mathrm{M}$ (black curve) was incubated with A549 cells for $24 \mathrm{~h}$. Expression levels of MICB and ULBP1 on the surface of A549 cells were significantly enhanced compared with those before the use of erlotinib $(\mathrm{P}<0.05)$. MICA expression decreased compared with that before the use 
of erlotinib $(\mathrm{P}<0.05)$. ULBP2 and ULBP3 showed no significant changes. Between 5 and $10 \mu \mathrm{M}$ erlotinib, the NKG2D ligand expression showed no significant difference $(\mathrm{P}>0.05)$.

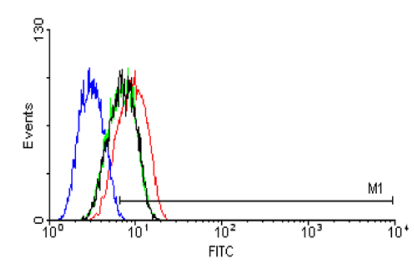

ULBP1

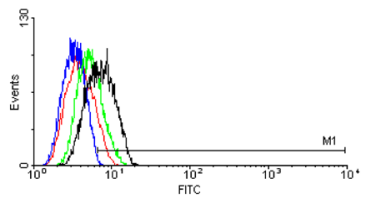

ULBP3

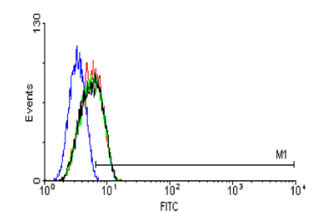

ULBP5

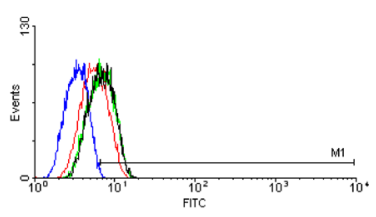

ULBP2

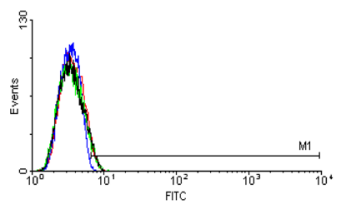

ULBP4

Figure 2. Analysis of NKG2D ligands expression in A549 cells following treatment with erlotinib.

\section{Effects of EGFR downstream molecules on NKG2D ligand expression in A549 cells}

Flow cytometry results showed that when STAT21 (STAT3 inhibitor) and SB203580 [mitogen-activated protein kinase (MAPK) inhibitor] were cultured with A549 cells after 24 $\mathrm{h}, \mathrm{NKG} 2 \mathrm{D}$ ligand expression on the A549 cell surface showed no significant change $(\mathrm{P}>0.05$; Table 1). LY294002 [phosphoinositide 3-kinase (PI3K) inhibitor] decreased surface MICA expression in A549 cells $(\mathrm{P}<0.05)$, but did not affect the expression of other ligands.

\begin{tabular}{|c|c|c|c|c|c|}
\hline Group & MICA & MICB & ULBPI & ULBP2 & ULBP3 \\
\hline Control & $66.62 \pm 1.41$ & $32.75 \pm 2.37$ & $9.80 \pm 2.40$ & $4.09 \pm 1.94$ & $34.96 \pm 4.22$ \\
\hline STAT21 & $67.37 \pm 0.54$ & $31.59 \pm 0.41$ & $10.58 \pm 0.86$ & $4.19 \pm 0.19$ & $32.86 \pm 0.35$ \\
\hline LY294002 & $42.90 \pm 3.49^{*}$ & $32.40 \pm 0.30$ & $9.58 \pm 1.22$ & $4.40 \pm 0.07$ & $34.65 \pm 0.35$ \\
\hline SB203580 & $66.08 \pm 0.28$ & $31.79 \pm 0.19$ & $9.15 \pm 0.39$ & $4.36 \pm 0.10$ & $34.97 \pm 0.40$ \\
\hline
\end{tabular}

$* \mathrm{P}<0.05$ vs control.

\section{Erlotinib enhanced the lethal sensitivity of CIK cells against A549 cells}

When the effector to target ratios were 10:1,20:1, and 30:1, the cytotoxicity values of CIK cells against A549 cells were $11.08 \pm 1.22,36.22 \pm 0.91$ and $45.73 \pm 2.00 \%$, respectively. 
The cytotoxicity values of CIK cells against A549 cells after addition of $10 \mu \mathrm{M}$ erlotinib were $18.24 \pm 0.96,48.49 \pm 0.78$ and $56.67 \pm 2.11 \%$, respectively. Using the same effector to target ratio, the A549 cell-killing activity of CIK cells was significantly enhanced after erlotinib addition $(\mathrm{P}<0.05)$. When the effector to target ratio was 20:1, NKG2D mAb closed CIK cell surface NKG2D receptor, the cytotoxicities of CIK cells on A549 cells before and after erlotinib use were $7.29 \pm 0.73$ and $7.99 \pm 0.11 \%$, respectively. The difference was not statistically significant $(\mathrm{P}>0.05)$.

\section{DISCUSSION}

The NKG2D-NKG2D ligand pathway plays an important role in anti-tumor immunity, with NKG2D as the main activating receptor of NK cells that induces anti-tumor effects (Bae et al., 2012a; Morisaki et al., 2012). There are 2 categories of NKG2D ligands (Bae et al., 2012a), including major histocompatibility complex class I chain-related molecule A or B (MICA, MICB) and human cytomegalovirus glycoprotein UL16 binding proteins (ULBP1, ULBP2, ULBP3). NKG2D ligands are widely expressed on the surface of tumor cells. The NKG2D receptor on the surface of immune cells and NKG2D ligands on the surface of tumor cells combine to stimulate the anti-tumor immune response.

CIK cells are heterogeneous cells cultivated by the co-culture of human peripheral blood mononuclear cells and a variety of cytokines in vitro. Compared with natural killer cells, the low expression of CIK cells inhibits immunoglobulin receptors. CIK cells express the NKG2D receptor (Mei et al., 2011) and play a role in anti-tumor effects (Franceschetti et al., 2009). CIK cells can be easily amplified in vitro for clinical applications (Laport et al., 2011; Mesiano et al., 2012). CIK cells are closely related to the cytotoxicity of tumor cells and tumor cell surface expression of NKG2D ligands (Mesiano et al., 2012). Studies have shown that chemotherapy drugs and molecular-targeted therapy can induce NKG2D ligand expression on the tumor cell surface and enhance the cytotoxic activity of immune cells through a variety of signaling pathways (Tang et al., 2008; Xu et al., 2011; Bae et al., 2012b). EGFR is overexpressed on the surface of various tumor cells and regulates the molecular activity of downstream signaling pathways, including the Ras-Raf-MAPK and PENT-PI3K-AKT pathways. In addition, JAK/STAT3 participates in the EGFR signaling pathway. These pathways are associated with tumor cell differentiation, proliferation, apoptosis, invasion, and angiogenesis. EGFR-targeted drugs have become a hot spot in the treatment of lung cancer in recent years. Lung adenocarcinoma A549 cells express wild-type EGFR. In this study, we used A549 cells.

NKG2D ligand expression is regulated by numerous factors. MICA expression is actively regulated by the STAT3, PI3K, and MAPK molecules (Molinero et al., 2003; Boissel et al., 2006; Bedel et al., 2011). In this study, we used the STAT3 inhibitor STAT21 and the MAPK inhibitor SB203580, which did not affect NKG2D ligand expression. However, the PI3K inhibitor LY294002 downregulated MICA expression on the surface of A549 cells. This indicated that erlotinib-induced MICA downregulation of A549 cells was associated with EGFR/PI3K signal suppression. MICB and ULBP1 upregulation was not associated with these molecules, indicating that EGFR signaling pathway regulation of MICB and ULBP1 expression requires further analysis.

Although MICA downregulation of A549 cells treated with erlotinib was observed, the killing activity of CIK cells increased significantly. When the NKG2D receptor on the CIK cell surface was blocked, the A549 cell killing activity of CIK cells showed no difference 
before and after treatment with erlotinib. The results indicate that the killing of CIK cells on A549 cells occurs through a combination of NKG2D receptor and NKG2D ligands. Erlotinib mainly increased activated ligand MICB and ULBP1 expression on the surface of A549 cells as well as enhanced sensitivity of A549 cells for CIK cell killing.

Based on our results, EGFR TKIs in vivo may stimulate the anti-tumor effects of immune cells. EGFR TKIs combined with CIK cells may be useful for adoptive immunotherapy of lung cancer.

\section{ACKNOWLEDGMENTS}

Research supported by the Zhengzhou Science and Technology Program Research Project (\#2010SFXM154).

\section{REFERENCES}

Bae DS, Hwang YK and Lee JK (2012a). Importance of NKG2D-NKG2D ligands interaction for cytolytic activity of natural killer cell. Cell. Immunol. 276: 122-127.

Bae JH, Kim SJ, Kim MJ, Oh SO, et al. (2012b). Susceptibility to natural killer cell-mediated lysis of colon cancer cells is enhanced by treatment with epidermal growth factor receptor inhibitors through UL16-binding protein-1 induction. Cancer Sci. 103: 7-16.

Bedel R, Thiery-Vuillemin A, Grandclement C, Balland J, et al. (2011). Novel role for STAT3 in transcriptional regulation of NK immune cell targeting receptor MICA on cancer cells. Cancer Res. 71: 1615-1626.

Boissel N, Rea D, Tieng V, Dulphy N, et al. (2006). BCR/ABL oncogene directly controls MHC class I chain-related molecule A expression in chronic myelogenous leukemia. J. Immunol. 176: 5108-5116.

Franceschetti M, Pievani A, Borleri G, Vago L, et al. (2009). Cytokine-induced killer cells are terminally differentiated activated CD8 cytotoxic T-EMRA lymphocytes. Exp. Hematol. 37: 616-628.

Han SW, Kim TY, Hwang PG, Jeong S, et al. (2005). Predictive and prognostic impact of epidermal growth factor receptor mutation in non-small-cell lung cancer patients treated with gefitinib. J. Clin. Oncol. 23: 2493-2501.

Huang Y, Wang Y, Li Y, Guo K, et al. (2011). Role of sorafenib and sunitinib in the induction of expressions of NKG2D ligands in nasopharyngeal carcinoma with high expression of ABCG2. J. Cancer Res. Clin. Oncol. 137: 829-837.

Inagaki A, Ishida T, Yano H, Ishii T, et al. (2009). Expression of the ULBP ligands for NKG2D by B-NHL cells plays an important role in determining their susceptibility to rituximab-induced ADCC. Int. J. Cancer 125: 212-221.

Laport GG, Sheehan K, Baker J, Armstrong R, et al. (2011). Adoptive immunotherapy with cytokine-induced killer cells for patients with relapsed hematologic malignancies after allogeneic hematopoietic cell transplantation. Biol. Blood Marrow Transplant 17: 1679-1687.

Linn YC, Yong HX, Niam M, Lim TJ, et al. (2012). A phase I/II clinical trial of autologous cytokine-induced killer cells as adjuvant immunotherapy for acute and chronic myeloid leukemia in clinical remission. Cytotherapy 14: 851-859.

Mei JZ, Guo KY, Wei HM, et al. (2007). In different tumor cell surface expression of MICA study and cytotoxic activity of NK cells. Zhong Guo Mian Yi Xue Za Zhi 23: 37-40.

Mei JZ, Liu GJ, Feng RT and Guo KY (2009). Cisplatin enhanced expression and activity of NK cells is enhanced role of NKG2D ligands in nasopharyngeal carcinoma cells. Zhong Liu Fang Zhi Yan Jiu 36: 996-998.

Mei YZ, Liu GJ and Li RJ (2011). IL-15 up-regulated the expression of NKG2D enhanced cell killing activity of CIK esophageal cancer EC9706 cells. Zhong Liu Fang Zhi Yan Jiu 38: 243-245.

Mesiano G, Todorovic M, Gammaitoni L, Leuci V, et al. (2012). Cytokine-induced killer (CIK) cells as feasible and effective adoptive immunotherapy for the treatment of solid tumors. Expert Opin. Biol. Ther. 12: 673-684.

Molinero LL, Fuertes MB, Fainboim L, Rabinovich GA, et al. (2003). Up-regulated expression of MICA on activated T lymphocytes involves Lck and Fyn kinases and signaling through MEK1/ERK, p38 MAP kinase, and calcineurin. $J$. Leukoc. Biol. 73: 815-822.

Morisaki T, Onishi H, Koya N, Tanaka H, et al. (2011). Combinatorial cytotoxicity of gemcitabine and cytokine-activated killer cells in hepatocellular carcinoma via the NKG2D-MICA/B system. Anticancer Res. 31: 2505-2510.

Morisaki T, Onishi H and Katano M (2012). Cancer immunotherapy using NKG2D andDNAM-1 systems. Anticancer Res. 32: 2241-2247. 
Rho JK, Choi YJ, Choi YR, Kim SY, et al. (2011). The effect of acquired cisplatin resistance on sensitivity to EGFR tyrosine kinase inhibitors in EGFR mutant lung cancer cells. Oncol. Res. 19: 471-478.

Tang KF, Ren H, Cao J, Zeng GL, et al. (2008). Decreased Dicer expression elicits DNA damage and up-regulation of MICA and MICB. J. Cell. Biol. 182: 233-239.

Xu X, Rao GS, Groh V, Spies T, et al. (2011). Major histocompatibility complex class I-related chain A/B (MICA/B) expression in tumor tissue and serum of pancreatic cancer: role of uric acid accumulation in gemcitabine-induced MICA/B expression. BMC Cancer 11: 194. 